care plans and one VA medical center. Results: Subjects in the study groups had similar baseline demographic and health characteristics. Sixty percent of patients offered $\mathrm{HaH}$ care opted for it $(\mathrm{n}=84)$. Patients in $\mathrm{HaH}$ received types of treatments associated with hospital care. Length of stay was shorter for patients in $\mathrm{HaH}, 3.2$ vs. 4.9 d, $(P=0.001)$. Patients cared for in $\mathrm{HaH}$ had reduced risk of clinical complications including incident delirium, adjusted odds ratio $0.26(0.12,0.57)$ and use of sedative medications, adjusted odds ratio $0.49(0.30,0.81)$. HaH care was associated with trends towards better physical functional outcomes. Illness-specific quality of care standards were met in similar proportions in $\mathrm{HaH}$ and hospital-treated groups. Patient and caregiver satisfaction with $\mathrm{HaH}$ was better than that associated with hospital care across multiple domains $(P<0.001)$. Family members of $\mathrm{HaH}$ patients experienced significantly less stress related to the caring for an acutely ill family member during the $\mathrm{HaH}$ episode compared with patients treated in the hospital $(P<0.001)$. Costs of $\mathrm{HaH}$ care were lower for $\mathrm{HaH}$ patients. Conclusions: $\mathrm{HaH}$ is an efficacious model of acute care delivery for selected older persons with certain medical illnesses. Dissemination of the $\mathrm{HaH}$ model into managed care settings is warranted.

\section{Abstract PS1-48}

A Team Approach to Improving Post Hip Fracture Care

Veronica Piziak, MD, PhD, Scott \& White; Hasan Rajab, PhD, Scott \& White; Jonathan Minor, MD, Scott \& White/Texas A\&M Health Science Center

Background: Patients over the age of 50 sustaining hip fractures by falling only from a standing height are at high risk for osteoporosis and recurrent fracture. Unfortunately, this group frequently leaves the hospital without any therapy to prevent future fractures. A previous survey of 183 hip fracture patients reviewed discharges for the years 1998-2000 at Scott \& White Memorial Hospital and revealed an area of concern. Of this group only 48 subjects $(26 \%)$ had a prior diagnosis of osteoporosis, but only $34(19 \%)$ received bisphosphonates and $29(16 \%)$ received hormone replacement therapy sometime during the 1 year follow-up period. This abstract describes the results of an initiative to increase the therapeutic interventions for osteoporosis in the post hip fracture patients at the Scott \& White facility of the Texas A\&M Health Science Center. Methods: A clinical database was established at Scott and White to track this population. The endocrinology and orthopedic areas agreed to add calcium supplements (1500 mg daily) containing vitamin D (400 IU daily), a DEXA scan request and a consult with endocrinology to the hospital discharge orders in appropriate patients. Three hundred fifty-five patients were enrolled in the database between September 2004 and June 2006. One hundred sixty-six patients who were followed for at least 6 months and who qualified for osteoporosis medication were used for the analysis. Results: Thirty-six (26.3\%) patients used calcium supplements with vitamin D before their fracture compared to $91(66.43 \%)$ of patients at follow-up $(P<0.001)$. Seventeen subjects $(12.41 \%)$ were taking bisphosphonates before their fracture and the number increased to 66 $(48.18 \%)$ after the treatment intervention. Eighty-two patients $(49.6 \%, P$ $<0.001)$ kept the appointment for the DEXA scan and $54(32.5 \%)$ saw the endocrinologist. Conclusions: The addition of calcium supplement with vitamin $\mathrm{D}$ to the hospital discharge medications was an effective and cost efficient method of improving treatment and enhancing compliance over the study period. Providing the opportunity for a routine endocrinology outpatient consultation after hip fracture increased the treatment of osteoporosis with effective bone active medication; however, many of the patients did not attend the consult.

Abstract PS1-49

Development, Implementation and Evaluation of a Web-based Curriculum on Pharmaceutical Marketing and Prescribing

April L. Salisbury, MBA, Lovelace Clinic Foundation; Margaret J. Gunter, $\mathrm{PhD}$, Lovelace Clinic Foundation; Sara J. Beaton, PhD, Lovelace Clinic Foundation

Background/Aims: To use grant funding resulting from lawsuit settlement funds from off-label marketing of Neurontin ${ }^{\circledR}$ to develop, implement, and evaluate a web-based curriculum on drug marketing and prescribing, and to revise the material using evaluation data from a live pilot session. The final revised curriculum will be made available to prescribers in the public domain. Methods: Content and education experts developed an educational course entitled 'Optimal Prescribing,' which consisted of five modules of slides, video clips, narration, cases, interviews, and interactive slides. The course was presented to physicians, physician assistants, and nurse practitioners from the Lovelace Health System in Albuquerque, New Mexico, in a live 5-hour workshop. Data were collected using an audience response system, a pretest and posttest, a written evaluation form, and staff observations. These data were analyzed and summarized to revise the material to produce web-based modules for use by prescribers in the public domain. In addition to the live pilot evaluation, these prescribers and a control group of providers who did not attend the pilot course will be surveyed to assess the impact of the education on attitudes about relationships with industry representatives, the amount and source of information that guide prescribing decisions, and self-reported changes in prescribing patterns. Results: Twenty-five prescribers participated in the live pilot session on June 23, 2007. From the analysis of the data, it is clear that the materials must be reduced in length and tied to very clear learning objectives in order for future users of the material to be motivated to complete the course. The longer the module in the pilot session, the less engaged the learners were. The learners expressed a need for more information on interpreting research studies and performing calculations such as the number needed to treat/harm. The learners valued a checklist of information needed to make informed prescribing decisions and lists of balanced sources of information. The 6 month post-pilot survey is being prepared and results will be available by March 2008. Conclusions: To make this curriculum more useful to prescribers, it must be simplified in format and content, while preserving and enhancing the key insights of the curriculum. Subsequent evaluation will include the self-reported effect on prescribing and attitudes toward industry influence on prescribing decisions.

\section{Abstract PS1-50 \\ Format Change of Computerized Test Order Form Affects Physician Behavior}

Varda Shalev, MD, MHA, Maccabi Healthcare Services, Israel; Gabriel Chodick, PhD, Maccabi Healthcare Services, Sackler Faculty of Medicine, Tel Aviv University; Lena Rosenmann, MSc, Maccabi Healthcare Services, Israel; Anat Beit-Or, PhD, Maccabi Healthcare Services, Israel; Virginia Sima, MSc, Maccabi Healthcare Services, Israel; Anthony D. Heymann, BSc MB BS MRCGP MHA, Maccabi Healthcare Services, Sackler Faculty of Medicine, Tel Aviv University, Israel

Background: Inappropriate use of laboratory diagnostic tests lowers quality and raises costs of care. Our study was designed to find whether a reduction in testing could be obtained by the simple strategy of changing the set-up of the check-box laboratory order form that is embedded in a computerized medical record. Methods: A prospective intervention study was carried out at Maccabi Healthcare Services (MHS), an Israeli HMO that has used a computerized medical record since 1992. The MHS central data repository retains complete historical records of patient demographic data, physician data, laboratory results, using the patient's unique national identification number. In May 2005 we changed the order form and reduced the number of tests that can be ordered from 51 to 26 using a check-box form. Twenty-seven tests were removed from the form and two tests were added. Changes in physician' test ordering were then studied. Results: While tests that were added or retained on the computerized laboratory order form showed an increase of $17 \%$ and $64 \%$ in number of tests ordered per visit compared to the period prior to intervention, tests that were removed showed a reduction of $7 \%$ in test ordering and a further reduction of $28 \%$ in the subsequent year. Conclusions: Changes in format of laboratory test order forms may be a powerful tool to improve appropriateness of testing. A thoughtfully built test ordering form can save unnecessary tests and can reinforce clinical guidelines for the performance of some preventive testing and follow-up. Electronic health records can be utilized for implementing policy. 